\title{
Early mobilisation of patients in the acute hospital setting following aneurysmal subarachnoid haemorrhage - a survey of current physiotherapy practice
}

Sabrina Hernandez BPhysio, GDipNR

Senior Physiotherapist, Alfred Health, Melbourne, Victoria, Australia

Peter Thomas BPhty (Hons), PhD, FACP

Consultant Physiotherapist and Team Leader - Critical Care and Surgery, Department of Physiotherapy, Royal Brisbane and Women's Hospital

Andrew Udy $M B C h B, F C I C M, P h D$

Head of Trauma ICU, The Alfred ICU \& Co-Deputy Director, Australian and New Zealand Intensive Research Centre, Monash University, Melbourne, Victoria, Australia

Carol Hodgson PhD, FACP, BAppSC(Physio)

Co-Deputy Director, Australian and New Zealand Intensive Research Centre, Monash University, Senior Physiotherapist, Intensive Care, Alfred Health, Melbourne, Victoria, Australia

\section{ABSTRACT}

Aneurysmal subarachnoid haemorrhage is a catastrophic form of stroke. There is very limited literature to guide physiotherapists on the type and timing of mobility interventions that should be provided during the acute phase. The aim of this study was to determine the current practices of physiotherapists in early mobilisation of patients with aneurysmal subarachnoid haemorrhage. A purposedesigned electronic survey was distributed to 71 physiotherapists in hospitals that specialise in the management of aneurysmal subarachnoid haemorrhage throughout Australia and New Zealand. A response rate of $80 \%$ was obtained $(n=57)$. Prior to the aneurysm being repaired, the most common practice reported by physiotherapists was not to mobilise patients (41\%). Once the aneurysm was repaired, mobility goals increased with $>80 \%$ of physiotherapists reporting goals of sitting on the edge of the bed or step transferring to a chair day one post repair. Physiotherapists reported that vasospasm, delayed cerebral ischaemia, recent further bleed, hypotension or the use of high level of noradrenaline would prevent them from mobilising patients. Only four respondents reported that they had a mobilisation protocol for aneurysmal subarachnoid haemorrhage patients at their hospital. Further research is required into the safety, timing and efficacy of early mobilisation practices in the management of aneurysmal subarachnoid haemorrhage patients.

\section{Hernandez, S., Thomas, P., Udy, A., Hodgson, C. (2018). Early mobilisation of patients in the acute hospital setting following aneurysmal subarachnoid haemorrhage - a survey of current physiotherapy practice. New Zealand Journal of Physiotherapy 46(3): 113-132. doi:10.15619/NZJP/46.3.04}

Keywords: Physiotherapy, Subarachnoid haemorrhage, Physical therapy modalities, Intensive care, Survey

\section{INTRODUCTION}

Subarachnoid haemorrhage (SAH) accounts for approximately $5 \%$ of all strokes, with $85 \%$ of SAHs resulting from aneurysm rupture (Luoma \& Reddy, 2013). Aneurysmal subarachnoid haemorrhage (aSAH) is a catastrophic event, with mortality rates being reported as high as $39-67 \%$ (The ACROSS Group, 2000; Nieuwkamp et al., 2009). Aneurysms are thought to form due to haemodynamic stress at cerebral arterial bifurcations leading to a dilatation of the vessel wall (Raya \& Diringer, 2014). Aneurysmal subarachnoid haemorrhage commonly occurs in people aged 45 - 64 years old, when patients are leading productive and independent lives (Lai \& Morgan, 2012). The potential physical, cognitive and psychosocial deficits commonly associated with aSAH often prevent patients returning to their previous level of function, severely impacting on their long-term quality of life (Saciri \& Kos, 2002).

Patients admitted with aSAH are frequently faced with a complicated recovery period in hospital, which involves prolonged monitoring. Common complications following aSAH include re-bleeding, vasospasm and delayed cerebral ischaemia (DCI) (Diringer et al., 2011; Suarez, 2015). Currently, there is very limited literature to guide physiotherapists on the timing and type of mobility interventions that should be provided during the acute period following the bleed. Furthermore, progressing patients through higher levels of mobilisation and the effects on cerebral perfusion are not known. Although early mobilisation guidelines are not integrated into the current recommendations from the Neurocritical Care Society (Diringer et al., 2011) and American Heart Association Stroke Council (Connolly et al., 2012), there has been recent evidence demonstrating that it is safe and feasible (Karic et al., 2015; O'Shea \& Stiller, 2016; Olkowski et al., 2013). The aim of this study was to determine the current practices of physiotherapists in early mobilisation of patients with aSAH and to report physiotherapists' perceived risks and barriers to early mobilisation. 


\section{METHODS}

Ethical approval for the study was obtained through relevant human research ethics committees.

A purpose-designed electronic survey was undertaken. The survey was designed to determine current early mobilisation practices following aSAH as there were no validated tools for benchmarking mobilisation in this setting. The survey questions were developed collaboratively by the investigators, who had extensive knowledge and clinical experience in aSAH from medical or physiotherapy backgrounds in tertiary hospital settings. For the purpose of this study, mobilisation was defined as sitting on the edge of the bed, sitting out of bed, step transferring or ambulation. This questionnaire comprised of 36 questions and was divided into three parts - Part A General questions, Part B - ICU related questions, and Part C - Neurosurgery ward specific questions. (See Appendix 1). The survey was pilot-tested by seven senior physiotherapists from two major acute hospitals in Victoria and Queensland. Feedback on the survey was obtained regarding question design, structure and content. As a result of the feedback, minor changes were made.

All respondents were asked to respond to questions in Part A. Physiotherapists then had the option of answering questions based on their main area of clinical practice, which could include ICU only, neurosurgical wards only, or both ICU and ward questions. The questions sought information regarding demographic characteristics of the respondents, characteristics of the physiotherapy service, potential risks to mobility, the timing, frequency and type of mobility and exercise interventions provided to patients and perceived barriers to mobility.
The survey was administered between August 2017 and January 2018. It was distributed to 39 hospitals in Australia and New Zealand that specialise in the management of aSAH via dedicated neurosurgical services, with potential sites identified from previous research (Udy et al., 2017). Physiotherapy managers in each centre were contacted via email and requested to forward the contact details of their senior physiotherapists in ICU and neurosurgery who were involved in the care of aSAH patients at their institution. Through this process, the survey was distributed electronically via SurveyMonkey (SurveyMonkey Inc.) and included the study invitation and information sheet. Completion of the online survey was considered consent to participate. Participants were able to withdraw any information provided at any time. Individual hospitals and participants were de-identified for analysis.

\section{Statistical Analysis}

The majority of the data was in the ordinal or nominal form and analysed in Excel (Microsoft Corporation). Open question data were analysed and grouped according to themes.

\section{RESULTS}

\section{Response Rate}

A total of 71 physiotherapists from 26 sites were identified and electronic access to the survey was provided. Figure 1 illustrates the flow of participants through the study. The response rate was $80 \%$ ( $n=57 / 71)$, however five participants only partially completed Part A of the survey. Thirty-three physiotherapists indicated they had ICU experience and completed Part B of the survey, and 35 had ward experience and were able to complete Part C. Results are provided as the number and percentage of total respondents to each question.

Information sent to physiotherapy

managers at 39 hospitals in Australia and

New Zealand specialising in aSAH

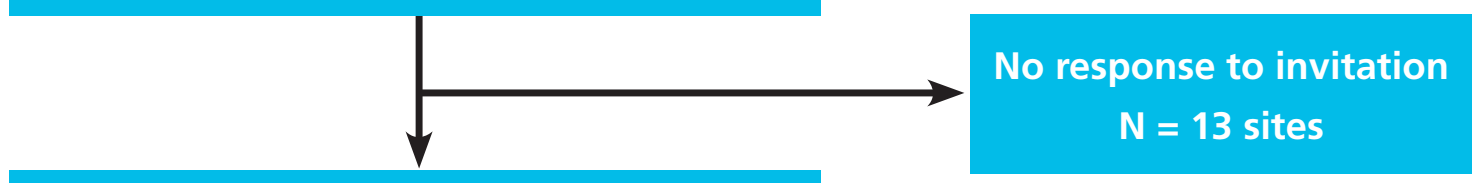

71 physiotherapists identified from

26 sites and invited to participate

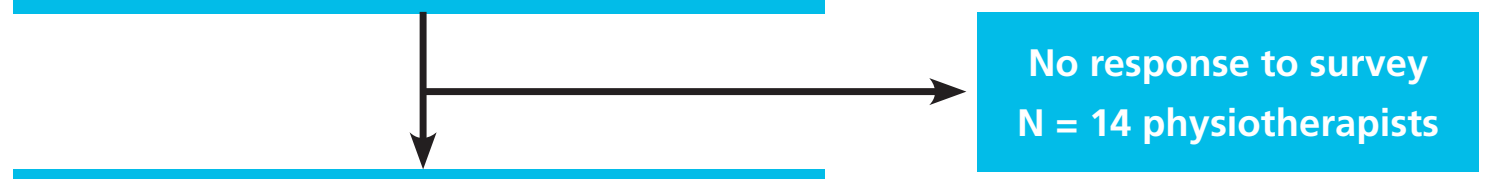

57 physiotherapists responded to survey

Figure 1: Flow of participants through study 
Characteristics of respondents and hospitals

Table 1 summarises the characteristics of the respondents. The majority of respondents ( $n=51 / 57,89 \%)$ were from Australia and six (11\%) were from New Zealand. Level of experience is shown in Table 1 with $74 \%$ of respondents being experienced physiotherapists with five or more years of experience working with aSAH patients. The vast majority of respondents worked in a public hospital setting $(n=52 / 57,91 \%)$ with the remainder working in a private hospital setting.

The two most frequently used aSAH grading scales were reported to be the World Federation of Neurosurgeons scale (Rosen \& Macdonald, 2005) ( $n=29 / 57,51 \%)$ and the Fisher scale (Rosen \& Macdonald, 2005) ( $n=20 / 57,35 \%)$. Thirty-nine percent $(n=22 / 57)$ of physiotherapists reported that they were unsure of which aSAH grading scale was used at their hospital.

Mobilisation prior to repair of the ruptured aneurysm Prior to the aneurysm being repaired, the most common practice reported by physiotherapists was not to mobilise patients (41\%, Figure 2). Sitting up in bed was reported by some respondents (30\%). Forty percent of physiotherapists on the ward would initiate sitting up in bed without medical consultation, this was also reflected in the ICU setting (37\% of responses). However, mobilisation at higher levels had a greater requirement for gaining medical approval. For example, on the ward approval from the neurosurgical team was required when sitting on the edge of bed (78\%), performing step transfers (86\%) and walking (100\%). In ICU, physiotherapists indicated they rarely commenced higher levels of mobilisation without the approval of a doctor with the neurosurgeons being more commonly consulted than the senior ICU doctors (64\% versus 36\%).

Mobilisation after repair of the ruptured aneurysm The timing of mobilisation of aSAH patients after a ruptured aneurysm has been repaired is summarised in Figure 3. For all sitting activities and step transfers to a chair, the majority of physiotherapists (>80\%) reported mobilising patients the first day after a ruptured aneurysm has been secured. A decline was seen for the goal of ambulation, with only $68 \%$ reporting this as being achieved day one post repair.

Type and frequency of mobility interventions

Only four respondents indicated that they had a mobilisation protocol for aSAH patients at their hospital. The majority of ICU physiotherapists ( $n=26 / 33,79 \%$ ) reported that patients with moderate to severe functional limitations would routinely be seen once a day in the ICU setting for mobilisation. The frequency of daily reviews appeared lower for patients with moderate to severe functional limitations who were on the

Table 1: Characteristics of the respondents

\begin{tabular}{|c|c|c|}
\hline & Response & $\mathrm{n}(\%$ of total) \\
\hline Location of work & VIC & $17(30)$ \\
\hline \multirow[t]{8}{*}{$(n=57)$} & TAS & $3(5)$ \\
\hline & NSW & $13(23)$ \\
\hline & QLD & $7(12)$ \\
\hline & WA & $3(5)$ \\
\hline & $\mathrm{ACT}$ & $2(4)$ \\
\hline & SA & $5(9)$ \\
\hline & NT & $1(2)$ \\
\hline & $\mathrm{NZ}$ & $6(11)$ \\
\hline Clinical experience in the & $<1$ year & $2(4)$ \\
\hline management of aSAH & $1-4$ years & $13(23)$ \\
\hline \multirow[t]{2}{*}{$(n=57)$} & $5-10$ years & $24(42)$ \\
\hline & $>10$ years & $18(32)$ \\
\hline Practice setting & Neurosurgery wards only & $20(35)$ \\
\hline \multirow[t]{4}{*}{$(n=57)$} & General ICU only & $24(42)$ \\
\hline & Neurosciences ICU only & $1(2)$ \\
\hline & General ICU and neurosurgery wards & $7(12)$ \\
\hline & Neurosciences ICU and neurosurgery wards & $5(9)$ \\
\hline
\end{tabular}

Notes: ICU, Intensive Care Unit; aSAH, aneurysmal subarachnoid haemorrhage; VIC, Victoria; TAS, Tasmania; NSW, New South Wales; QLD, Queensland; WA, Western Australia; ACT, Australian Capital Territory; SA, South Australia; NT, Northern Territory; NZ, New Zealand 


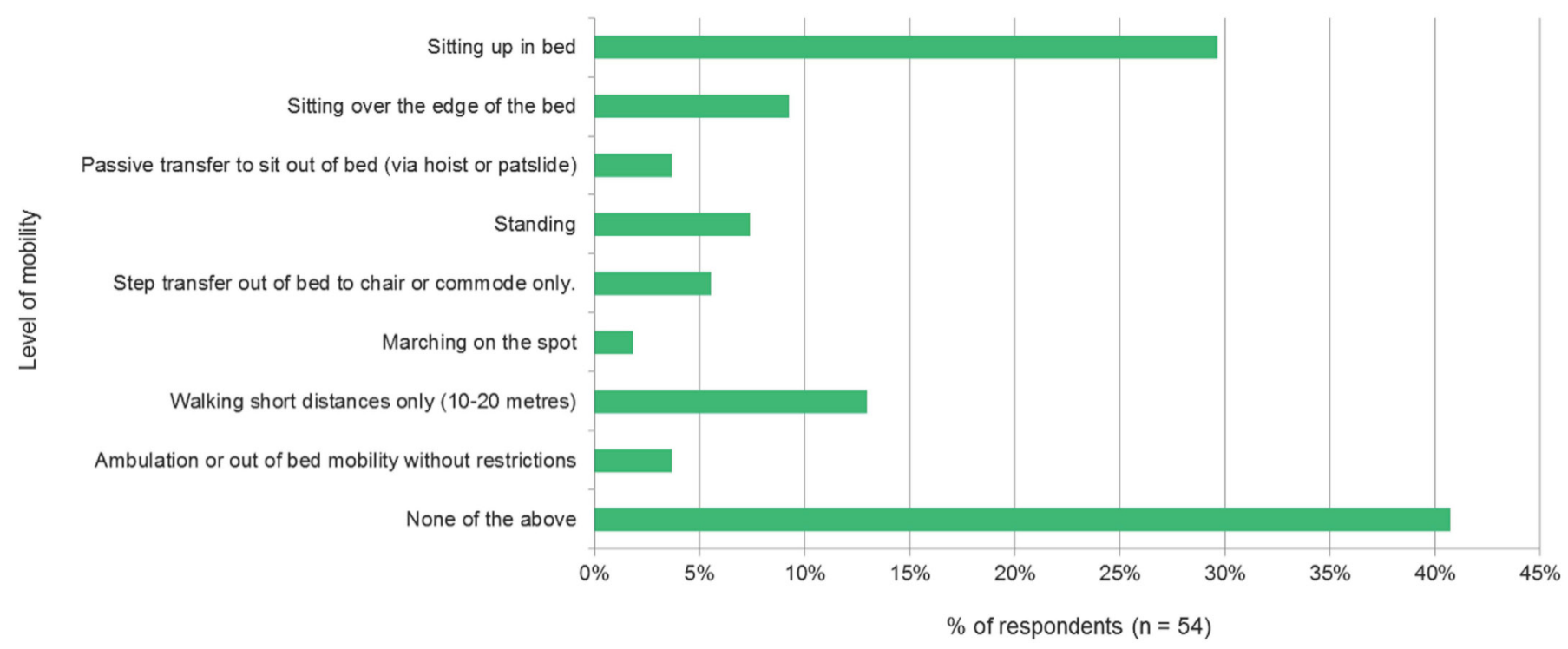

Figure 2: Level of mobility physiotherapists reported to be achieved prior to the ruptured aneurysm being repaired either by surgical clipping or endovascular coiling

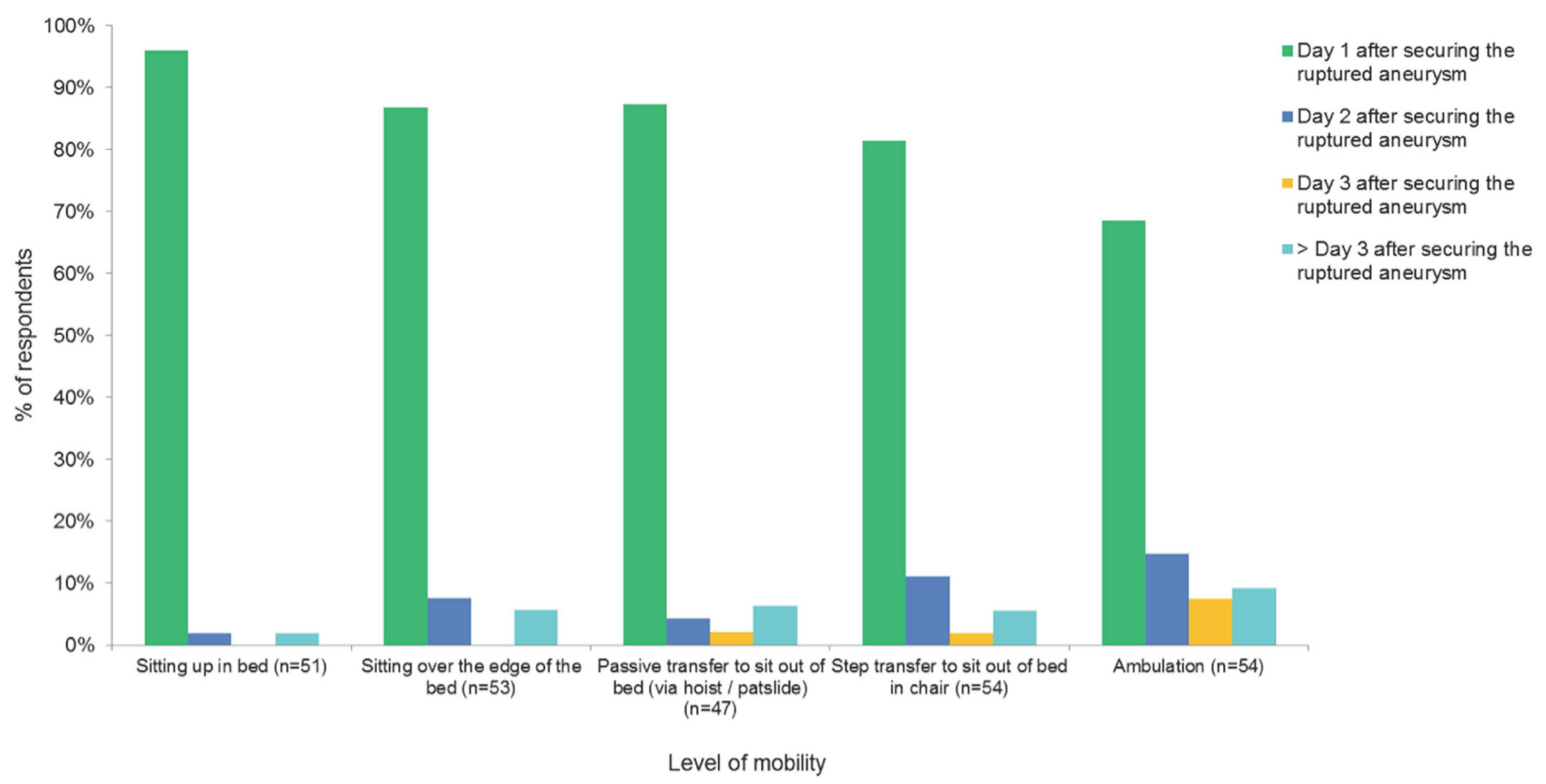

Figure 3: The first day aSAH patients were reported to be mobilised after the ruptured aneurysm has been repaired 


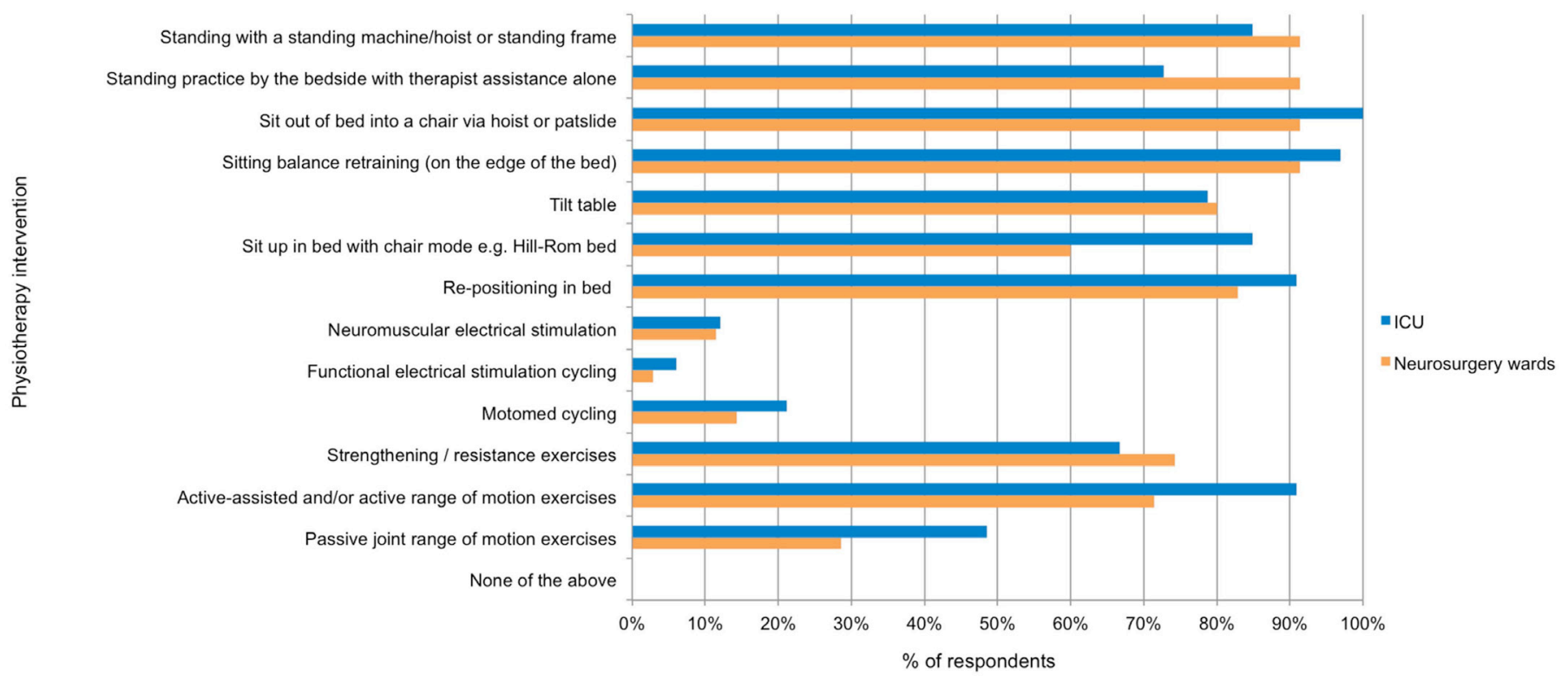

Figure 4: Physiotherapy interventions reported to be provided to aSAH patients with moderate to severe functional limitations in the ICU and neurosurgery ward settings

ward $(n=16 / 35,46 \%)$ and patients with mild or no functional limitations who were in ICU ( $n=14 / 33,42 \%)$ or the ward $(n=11 / 35,31 \%)$.

The types of physiotherapy interventions provided to aSAH patients with moderate to severe functional limitations in both the ICU and ward settings are summarised in Figure 4. In ICU, the most frequently reported type of physiotherapy interventions provided to patients with mild or no functional limitations included ambulation practice $(n=32 / 33,97 \%)$ and standing or dynamic balance practice $(n=24 / 33,73 \%)$. The most commonly reported interventions provided to these patients in the neurosurgery ward setting were similar, with $91 \%(n=32 / 35)$ undertaking ambulation practice and $89 \%(n=31 / 35)$ standing or dynamic balance practice.

Monitoring during mobilisation and perceived risks The majority of physiotherapists reported that they would typically monitor systolic blood pressure $(n=51 / 52,98 \%)$, level of consciousness ( $n=50 / 52,96 \%)$, headache $(n=47 / 52,90 \%)$, heart rate $(n=46 / 52,88 \%)$, percutaneous oxygen saturations $(n=44 / 52,85 \%)$ and upper and lower limb strength $(n=37 / 52$, $71 \%$ ) as patients were moved into more upright positions.

In regard to neurological risks to mobilisation (refer to Table 2), the majority of physiotherapists reported that they would prefer patients to remain in bed when vasospasm is present $(n=38 / 51$, $75 \%), \mathrm{DCl}(\mathrm{n}=36 / 50,72 \%)$ or there has been a recent further bleed $(n=41 / 52,79 \%)$. There were very few physiotherapists that reported that they were happy to perform mobilisation when there was recent confirmation of vasospasm (12\% or less), acute clinical signs of $\mathrm{DCl}(16 \%$ or less), recent further bleed ( $8 \%$ or less) or recent seizures (10\% or less).

The three most frequently reported factors that would lead to the clinical decision of the physiotherapist not to clamp the extraventricular drain (EVD) for mobilisation were new signs of neurological deterioration $(n=48 / 52,92 \%)$, medical clearance from either neurosurgeon or intensivist not being given $(n=48 / 52,92 \%)$ and if intracranial pressure was greater than $20 \mathrm{mmHg}(\mathrm{n}=42 / 52,81 \%)$. Physiotherapists also frequently reported that high amounts of blood draining from the EVD ( $n=40 / 52,77 \%$ ) or large amounts (>15 millilitres/hour) of cerebral spinal fluid (CSF) being drained per hour $(n=40 / 52$, $77 \%$ ) would also prevent them mobilising patients.

Both ICU and ward-based physiotherapists answered separate questions in relation to cardiovascular risks to mobilisation relevant to their work setting (refer to Table 3 and Table 4). The vast majority of both ICU $(n=29 / 33,88 \%)$ and ward $(n=33 / 35$, $94 \%$ ) physiotherapists were happy to ambulate patients if the blood pressure was autoregulating and at the desired target pressure. ICU physiotherapists reported that they preferred patients to remain in bed with minimal activity when the following factors were present: noradrenaline greater than $20 \mathrm{mcg} / \mathrm{min}$ to maintain blood pressure above the set target ( $n=24 / 32,75 \%)$, oral nimodipine recently administered and blood pressure was below desired target pressure $(n=25 / 33$, $76 \%$ ), uncontrolled hypertension requiring antihypertensives $(n=25 / 33,76 \%)$ and hypotension with mean arterial blood pressure less than $65 \mathrm{mmHg}(\mathrm{n}=28 / 32,88 \%)$. The majority of ward physiotherapists reported that the presence of the following factors would prevent them from mobilising patients: uncontrolled hypertension requiring antihypertensive medications ( $n=25 / 35,71 \%$ ) or hypotension with mean arterial blood pressure less than $65 \mathrm{mmHg}(n=30 / 35,86 \%)$.

Institutional barriers to mobilisation

Frequent barriers to mobilisation of aSAH patients within the ICU and neurosurgery ward settings are summarised in Table 5. Barriers to mobilisation were reported to be most common in patients with moderate to severe functional limitations with physiotherapists citing insufficient staffing and limited appropriate seating as the most frequent barriers in both the ICU and ward settings. 


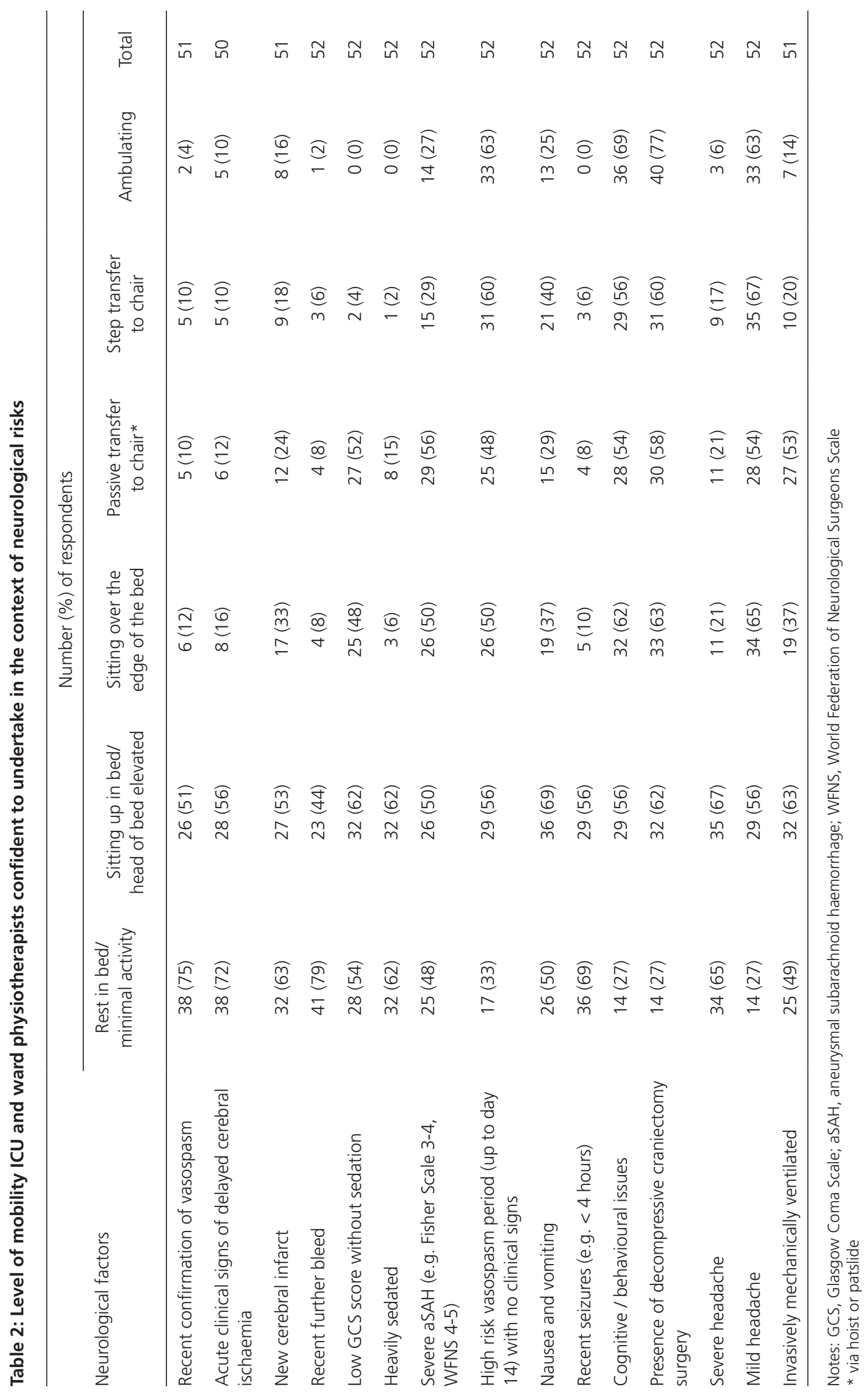




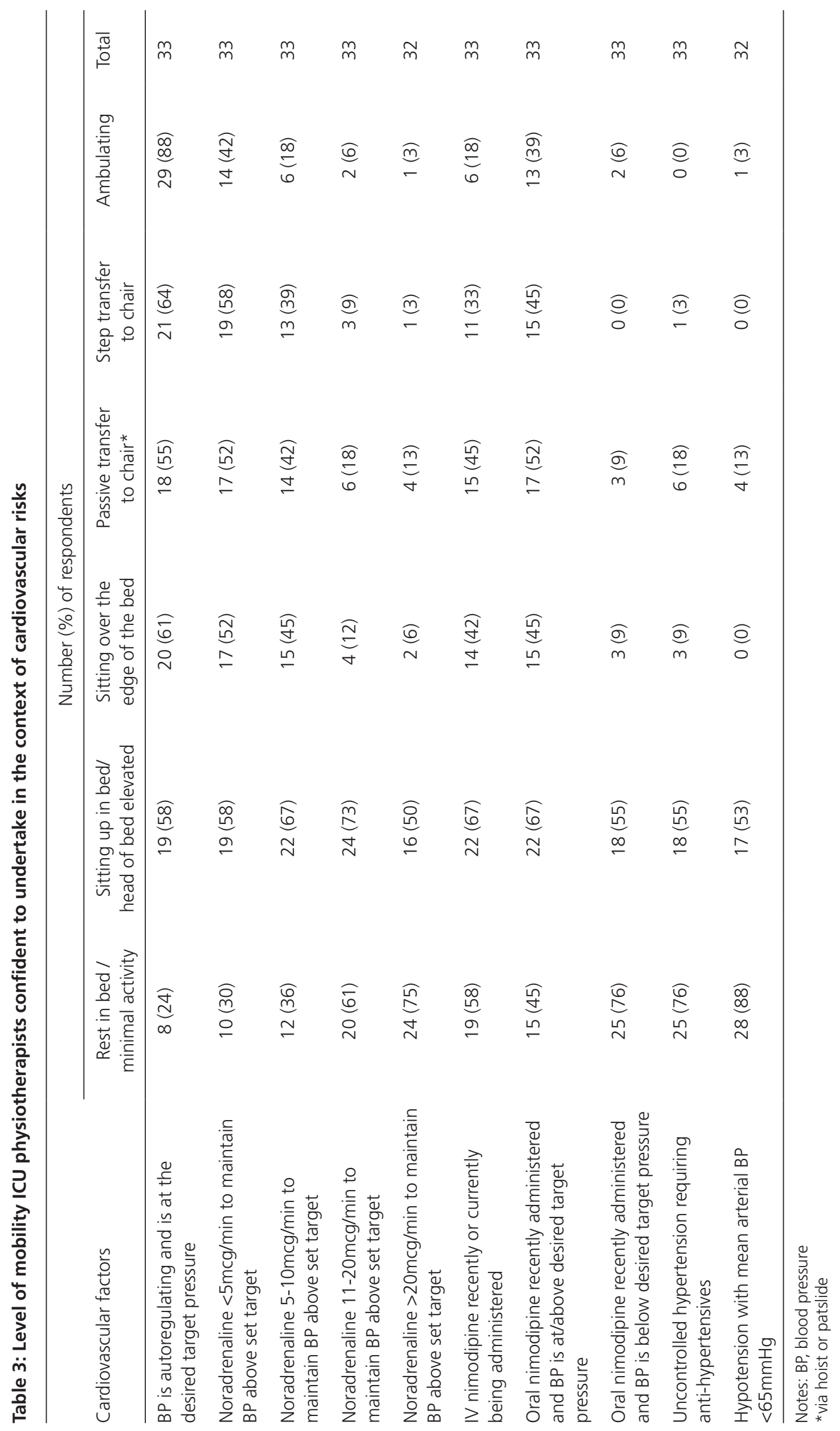


About two thirds of the respondents ( $n=36 / 52,69 \%$ ) reported that physiotherapists are not able to clamp the EVD in their institution, with the remainder $(n=16 / 52,31 \%)$ reporting that physiotherapists were able to clamp the EVD but with close liaison or supervision by nursing staff.

For patients with decompressive craniectomies, only 10\% of physiotherapists $(n=5 / 52$ ) reported that they would commence mobility without a helmet. Approximately a third of physiotherapists ( $n=19 / 52,37 \%$ ) reported that mobility would commence only once a helmet was fitted and 35\% ( $n=18 / 52)$ reported that a helmet was preferable but mobility could commence prior if there were other issues such as swelling or wound breakdown that would prevent its application.

\section{DISCUSSION}

This is the first study that has explored the decision making and mobilisation practices of physiotherapists from across multiple centres for patients with aSAH. In this sample of Australian and New Zealand centres, we found that few had established mobility protocols to guide mobilisation practices for patients with aSAH. Prior to an aneurysm being secured, physiotherapists were reluctant to initiate any level of mobility, except for sitting up in bed. As the risk of rebleeding from a ruptured cerebral aneurysm is very high, particularly during the initial period following the bleed, urgent medical management involves identifying the source of the bleed and repair of the ruptured aneurysm either by surgical clipping or endovascular coiling (Connolly et al., 2012; Diringer et al., 2011). To date, there is insufficient evidence to suggest that bedrest reduces the risk of mortality associated with rebleeding (Ma et al., 2013). This rebleeding risk and associated high risk of mortality are likely to explain the reluctance of physiotherapists to mobilise patients prior to the ruptured aneurysm being repaired. Almost all physiotherapists reported requiring neurosurgeon clearance if mobilisation were to occur prior to definitive management of the aneurysm. A conservative approach to mobilisation in this period may also be led by the symptoms patients often present with, including photophobia, severe headaches, neurological deficits, nausea and vomiting.

This study found that the majority of physiotherapists were happy to commence all levels of mobility the first day after the ruptured aneurysm had been secured. A strong drive for early mobilisation has evolved recently within the ICU environment (Tipping et al., 2017) with potential benefits demonstrated. However, recommendations for early mobilisation of aSAH patients are limited and potential harm has been found with early mobilisation of patients with stroke. The AVERT trial (Bernhardt et al., 2015) looked at early mobilisation of patients with stroke within 24 hours of stroke onset and found that it was associated with poorer functional outcomes at three months post-stroke. However, the AVERT trial did not include aSAH patients, or those in the ICU setting. There have been several small studies that have found early mobilisation to be safe and feasible in patients following aSAH in the ward (Karic et al., 2017; Karic et al., 2015) and ICU settings (O'Shea \& Stiller, 2016; Olkowski et al., 2013). Although these studies found a very low incidence of adverse events associated with early mobilisation, patients demonstrating a 
Table 5: Frequent* barriers to early mobilisation in the ICU and neurosurgery ward settings reported by physiotherapists

\begin{tabular}{|c|c|c|}
\hline \multirow[b]{2}{*}{ Barriers } & \multicolumn{2}{|c|}{ Number (\%) of respondents } \\
\hline & $\begin{array}{l}\text { ICU responses } \\
\quad(n=33)\end{array}$ & $\begin{array}{l}\text { Neurosurgery ward responses } \\
\qquad(\mathrm{n}=35)\end{array}$ \\
\hline $\begin{array}{l}\text { Insufficient staff to assist with mobilising patients with moderate to } \\
\text { severe functional limitations }\end{array}$ & $12(36)$ & $19(54)$ \\
\hline $\begin{array}{l}\text { Insufficient staff to assist with mobilising patients with mild or no } \\
\text { functional limitations }\end{array}$ & $6(18)$ & $5(14)$ \\
\hline $\begin{array}{l}\text { Insufficient staffing to monitor a high falls risk patient when sitting } \\
\text { out of bed }\end{array}$ & $13(39)$ & $12(34)$ \\
\hline $\begin{array}{l}\text { Limited access to appropriate seating/chairs to enable patients with } \\
\text { moderate to severe functional limitations to sit out of bed }\end{array}$ & $10(30)$ & $14(40)$ \\
\hline $\begin{array}{l}\text { Limited access to appropriate seating/chairs to enable patients with } \\
\text { mild or no functional limitations to sit out of bed }\end{array}$ & $4(12)$ & $1(3)$ \\
\hline $\begin{array}{l}\text { Limited access to transferring equipment (e.g. hoists, standing } \\
\text { machines or patslides) to enable patients with moderate to severe } \\
\text { functional limitations to sit out of bed. }\end{array}$ & $2(6)$ & $1(3)$ \\
\hline None of the above & $14(42)$ & $8(23)$ \\
\hline $\begin{array}{l}\text { Other (included limited gym space, lack of mobility protocol, lack of } \\
\text { standardisation of practice between neurosurgeons, rehabilitation } \\
\text { patients deprioritised) }\end{array}$ & $2(6)$ & $5(14)$ \\
\hline
\end{tabular}

Notes: * Frequent is defined as a barrier at least every second day

deterioration in neurological status or signs of vasospasm on screening were not mobilised. There have also been studies indicating early mobilisation to be associated with improved functional outcomes in older adults with low Hunt and Hess grades (Shimamura et al., 2014) and in a mixed intracerebral haemorrhage and aSAH patient population (Rand \& Darbinian, 2015), however, when Karic and colleagues (2016) examined the long-term effect of early rehabilitation, they found that there was no significant difference in functional outcomes at one year when an early mobilisation and control group were compared. It did however find that early mobilisation increased the chance of a good functional outcome in patients with aSAH who had high severity scores (WFNS grade 3-5).

Vasospasm of the cerebral blood vessels occurs in approximately two thirds of patients with aSAH between days three to 14 post-bleed (Macdonald, 2013). Around half of patients with vasospasm go on to develop a clinically detectable neurological deterioration termed $\mathrm{DCl}$ as a result of cerebral ischaemia (Connolly et al., 2012; Dabus \& Nogueira, 2013). Cerebral infarction can occur as a result of vasospasm and $\mathrm{DCl}$, and is strongly associated with poor functional outcomes (Frontera et al., 2009; Kreiter et al., 2009; Vergouwen et al., 2011). Medical treatment aims to provide early detection and prevention of cerebral ischaemia to reduce the risk of cerebral infarction (Diringer et al., 2011). From this study, it appears that overall, physiotherapists view the neurological complications of vasospasm and $\mathrm{DCl}$ as a contraindication to mobility.
Physiotherapists were also reluctant to mobilise patients if blood pressure was below the set target, patients were on a high level of noradrenaline or in the presence of uncontrolled hypertension. This was also reflected in reported practice where almost all (98\%) physiotherapists monitored systolic blood pressure as patients were moved into more upright positions. Despite this apprehension to mobilise patients with these risk factors, one prospective interventional study found that the risk of severe clinical vasospasm was significantly reduced with early mobilisation (Karic et al., 2017). This study did however report an increased use of intraarterial nimodipine to treat symptomatic vasospasm in the early mobility group. Another study by Riordan et al. (2015) also found that early mobilisation and mild exercise reduced the odds of patients developing symptomatic cerebral vasospasm. However, patients were analysed retrospectively from patient charts.

Patients admitted with aSAH often have delays in mobilisation due to poor neurological status, awaiting definitive management of the aneurysm or due to aSAH associated symptoms such as headache. It is well known from the literature that prolonged bedrest results in a number of complications that include reduced cardiac output, reduced vascular tone and venous pooling (Lee et al., 2010; Lee et al., 2014). These lead to a reduced ability to respond appropriately to orthostatic changes as patients move into more upright positions and could counteract the medical efforts to prevent DCI and cerebral ischaemia. Previous studies have looked at the effect of 
elevating the head of the bed in aSAH patients and found no significant change to cerebral flood flow (Blissitt et al., 2006; Kung et al., 2013). However the effects of higher levels of mobility such as sitting over the edge or standing on cerebral perfusion have not been investigated. The lack of evidence presents a dilemma to clinicians, in balancing the effects of immobility with the risk of secondary brain damage.

In this study, there were a number of potential institutional barriers to early mobilisation reported. Hospital practices that include fitting of helmets for patients following craniectomy surgery and the requirement of nursing staff to clamp the EVD may contribute to delays in mobilisation of patients in both the ward and ICU environments. In a study by Koo et al. (2016), the lack of hospital protocols and guidelines acted as a barrier to early mobilisation in the intensive care setting. In the current study, nearly all physiotherapists reported that they did not have mobility protocols at their hospital and this along with lack of evidence could have attributed to physiotherapists' reluctance to mobilise patients in the setting of perceived neurological and cardiovascular risks. Frequent barriers to mobilisation were most commonly reported in patients with moderate to severe functional impairments and similar to other studies were found to be due to insufficient staffing and lack of appropriate seating (Appleton et al., 2011; Koo et al., 2016).

There are several limitations to this study. Firstly, while we had 57 participants, these physiotherapists came from a smaller number of centres overall (26 of 39 neurosurgical centres). Therefore, more than one physiotherapist working in the same area at each hospital may have responded to the survey. However with the lack of mobility protocols in hospitals it is expected that individual physiotherapists within the same unit may have responded differently to the survey questions. Secondly, the survey is subject to responder bias and therefore a more accurate measure of current practice would be gained through an observational cohort study of patients with aSAH. Lastly, approximately two thirds of respondents answered either the ICU specific (Part B) or the neurosurgery ward specific questions (Part C), and hence not all respondents completed the entire survey due to the majority of physiotherapists having clinical expertise in only one of these clinical service areas.

This study provides important insight into reported early mobilisation practices of patients with aSAH and may enable physiotherapists to benchmark their practice against other specialised centres. This study has highlighted the need for further research into the timing and type of early mobilisation that is most effective in patients with aSAH. Furthermore, the effect of different levels of mobilisation on cerebral perfusion and neurological complications needs to be urgently investigated particularly in patients at high risk.

\section{CONCLUSION}

Physiotherapists in specialised centres reported early mobilisation of patients with aSAH once the ruptured aneurysm was repaired. However there are key perceived risks that prevented physiotherapists from mobilising patients that include vasospasm, $\mathrm{DCl}$, recent further bleed, blood pressure below the set target, uncontrolled hypertension and high levels of noradrenaline to maintain set blood pressure targets. There was variability in the type and frequency of exercises provided to stable patients with aSAH and there were also differences when comparing the ward and ICU settings. Insufficient staffing and limited access to appropriate seating were frequent barriers to mobilisation in patients with moderate to severe functional limitations. Furthermore, almost all physiotherapists reported that they did not have mobilisation protocols at their hospital. This study has highlighted the need for further research into the safety, timing and efficacy of mobility practices in the management of patients with aSAH particularly during the highrisk vasospasm period. This will enable the development of clear mobility protocols that can be used to guide best practice within Australian and New Zealand hospitals.

\section{KEY POINTS}

1. Physiotherapists reported that they did not mobilise patients if the ruptured aneurysm had not been repaired.

2. Almost all physiotherapists reported that they mobilise patients the first day after the ruptured aneurysm has been repaired.

3. Physiotherapists were concerned about mobilising patients if the following factors were present: vasospasm, delayed cerebral ischaemia, recent further bleed, hypotension or the use of high levels of noradrenaline.

4. The vast majority of physiotherapists reported that they did not have a mobilisation protocol at their hospital.

\section{DISCLOSURES}

No funding was obtained for this study. The authors declare no conflicts of interest.

\section{PERMISSIONS}

Ethics approval was obtained from the Alfred Health Ethics Committee (project 331/17) and South Eastern Sydney Local Health District Ethics Committee (project 17/LPOOL/437) as a low risk project.

\section{ACKNOWLEDGMENTS}

The authors wish to thank the Alfred Hospital Allied Health department for providing access to the SurveyMonkey software. The authors acknowledge Rodney Sturt, Jacqui Agostinello, Melissa Bowman, Christine James and Katie Acland who piloted the survey. The authors would also like to thank the physiotherapists who contributed their valuable time to completing the survey.

\section{ADDRESS FOR CORRESPONDENCE}

Sabrina Hernandez, Department of Physiotherapy, The Alfred Hospital, 55 Commercial Road, Melbourne, Victoria, Australia 3181. Telephone: +61 39076 3450. Email: s.hernandez@alfred. org.au

\section{REFERENCES}

The ACROSS Group (2000). Epidemiology of aneurysmal subarachnoid hemorrhage in Australia and New Zealand: incidence and case fatality from the Australasian Cooperative Research on Subarachnoid Hemorrhage Study (ACROSS). Stroke, 31(8), 1843-1850. 
Appleton, R. T., MacKinnon, M., Booth, M. G., Wells, J., \& Quasim, T. (2011). Rehabilitation within Scottish intensive care units: A national survey. Journal of the Intensive Care Society, 12(3), 221-227. doi:10.1177/175114371101200309.

Bernhardt, J., Langhorne, P., Lindley, R. I., Thrift, A. G., Ellery, F., Collier, J., ... Donnan, G. (2015). Efficacy and safety of very early mobilisation within $24 \mathrm{~h}$ of stroke onset (AVERT): a randomised controlled trial. Lancet, 386(9988), 46-55. doi:10.1016/s0140-6736(15)60690-0.

Blissitt, P. A., Mitchell, P. H., Newell, D. W., Woods, S. L., \& Belza, B. (2006). Cerebrovascular dynamics with head-of-bed elevation in patients with mild or moderate vasospasm after aneurysmal subarachnoid hemorrhage. American Journal of Critical Care, 15(2), 206-216.

Connolly, E. S., Jr., Rabinstein, A. A., Carhuapoma, J. R., Derdeyn, C. P., Dion, J., Higashida, R. T., ... Vespa, P. (2012). Guidelines for the management of aneurysmal subarachnoid hemorrhage: a guideline for healthcare professionals from the American Heart Association/ American Stroke Association. Stroke, 43(6), 1711-1737. doi:10.1161/ STR.0b013e3182587839.

Dabus, G., \& Nogueira, R. G. (2013). Current options for the management of aneurysmal subarachnoid hemorrhage-induced cerebral vasospasm: a comprehensive review of the literature. Interventional Radiology, 2(1), 3051. doi:10.1159/000354755.

Diringer, M. N., Bleck, T. P., Claude Hemphill, J., 3rd, Menon, D., Shutter, L., Vespa, P., . . . Zipfel, G. (2011). Critical care management of patients following aneurysmal subarachnoid hemorrhage: recommendations from the Neurocritical Care Society's Multidisciplinary Consensus Conference. Neurocritical Care, 15(2), 211-240. doi:10.1007/s12028-011-9605-9.

Frontera, J. A., Fernandez, A., Schmidt, J. M., Claassen, J., Wartenberg, K. E., Badjatia, N., . . Mayer, S. A. (2009). Defining vasospasm after subarachnoid hemorrhage: what is the most clinically relevant definition? Stroke, 40(6), 1963-1968. doi:10.1161/strokeaha.108.544700.

Karic, T., Roe, C., Nordenmark, T. H., Becker, F., \& Sorteberg, A. (2016). Impact of early mobilization and rehabilitation on global functional outcome one year after aneurysmal subarachnoid hemorrhage. Journal of Rehabilitation Medicine, 48(8), 676-682. doi:10.2340/16501977-2121.

Karic, T., Roe, C., Nordenmark, T. H., Becker, F., Sorteberg, W., \& Sorteberg, A. (2017). Effect of early mobilization and rehabilitation on complications in aneurysmal subarachnoid hemorrhage. Journal of Neurosurgery, 126(2), 518-526. doi:10.3171/2015.12.jns151744.

Karic, T., Sorteberg, A., Haug Nordenmark, T., Becker, F., \& Roe, C. (2015). Early rehabilitation in patients with acute aneurysmal subarachnoid hemorrhage. Disability and Rehabilitation, 37(16), 1446-1454. doi:10.310 9/09638288.2014.966162.

Koo, K. K., Choong, K., Cook, D. J., Herridge, M., Newman, A., Lo, V., Meade, M. O. (2016). Early mobilization of critically ill adults: a survey of knowledge, perceptions and practices of Canadian physicians and physiotherapists. Canadian Medical Association Journal Open, 4(3), E448-E454. doi:10.9778/cmajo.20160021.

Kreiter, K. T., Mayer, S. A., Howard, G., Knappertz, V., Ilodigwe, D., Sloan, M. A., \& Macdonald, R. L. (2009). Sample size estimates for clinical trials of vasospasm in subarachnoid hemorrhage. Stroke, 40(7), 2362-2367.

Kung, D. K., Chalouhi, N., Jabbour, P. M., Starke, R. M., Dumont, A. S., Winn, H. R., . . Hasan, D. M. (2013). Cerebral blood flow dynamics and head-of-bed changes in the setting of subarachnoid hemorrhage. BioMed Research International, 2013, 4. doi:10.1155/2013/640638.

Lai, L., \& Morgan, M. K. (2012). Incidence of subarachnoid haemorrhage: an Australian national hospital morbidity database analysis. Journal of Clinical Neuroscience, 19(5), 733-739. doi:10.1016/j.jocn.2011.09.001.

Lee, S. M., Moore, A. D., Everett, M. E., Stenger, M. B., \& Platts, S. H. (2010). Aerobic exercise deconditioning and countermeasures during bed rest. Aviation, Space, and Environmental Medicine, 81(1), 52-63.

Lee, S. M., Schneider, S. M., Feiveson, A. H., Macias, B. R., Smith, S. M., Watenpaugh, D. E., \& Hargens, A. R. (2014). WISE-2005: Countermeasures to prevent muscle deconditioning during bed rest in women. Journal of Applied Physiology (1985), 116(6), 654-667. doi:10.1152/japplphysiol.00590.2013.
Luoma, A., \& Reddy, U. (2013). Acute management of aneurysmal subarachnoid haemorrhage. Continuing Education in Anaesthesia Critical Care \& Pain, 13(2), 52-58. doi:10.1093/bjaceaccp/mks054.

Ma, Z., Wang, Q., \& Liu, M. (2013). Early versus delayed mobilisation for aneurysmal subarachnoid haemorrhage. Cochrane Database of Systematic Reviews(5), CD008346. doi:10.1002/14651858.CD008346.pub2.

Macdonald, R. L. (2013). Delayed neurological deterioration after subarachnoid haemorrhage. Nature Reviews Neurology, 10, 44 doi:10.1038/nrneurol.2013.246

Nieuwkamp, D. J., Setz, L. E., Algra, A., Linn, F. H., de Rooij, N. K., \& Rinkel, G. J. (2009). Changes in case fatality of aneurysmal subarachnoid haemorrhage over time, according to age, sex, and region: a metaanalysis. The Lancet Neurology, 8(7), 635-642. doi:10.1016/s14744422(09)70126-7.

O'Shea, K., \& Stiller, K. (2016). Early progressive mobilisation following acute subarachnoid or subdural haemorrhage: An observational pilot study. International Journal of Therapy and Rehabilitation, 23(7), 339-346. doi:10.12968/ijtr.2016.23.7.339.

Olkowski, B. F., Devine, M. A., Slotnick, L. E., Veznedaroglu, E., Liebman, K. M., Arcaro, M. L., \& Binning, M. J. (2013). Safety and feasibility of an early mobilization program for patients with aneurysmal subarachnoid hemorrhage. Physical Therapy, 93(2), 208-215. doi:10.2522/ptj.20110334.

Rand, M. L., \& Darbinian, J. A. (2015). Effect of an evidence-based mobility intervention on the level of function in acute intracerebral and subarachnoid hemorrhagic stroke patients on a neurointensive care unit. Archives of Physical Medicine and Rehabilitation, 96(7), 1191-1199. doi:10.1016/j.apmr.2015.02.008.

Raya, A. K., \& Diringer, M. N. (2014). Treatment of subarachnoid hemorrhage. Critical Care Clinics, 30(4), 719-733. doi:10.1016/j. ccc.2014.06.004

Riordan, M. A., Kyle, M., Dedeo, C., Villwock, M. R., Bauer, M., Vallano, M. L., \& Deshaies, E. M. (2015). Mild exercise reduces cerebral vasospasm after aneurysm subarachnoid hemorrhage: a retrospective clinical study and correlation with laboratory investigation. Acta Neurochirurgica Supplement, 120, 55-61.

Rosen, D. S., \& Macdonald, R. L. (2005). Subarachnoid hemorrhage grading scales: a systematic review. Neurocritical Care, 2(2), 110-118. doi:10.1385/ NCC: $2: 2: 110$

Saciri, B. M., \& Kos, N. (2002). Aneurysmal subarachnoid haemorrhage: outcomes of early rehabilitation after surgical repair of ruptured intracranial aneurysms. Journal of Neurology, Neurosurgery and Psychiatry, 72(3), 334-337.

Shimamura, N., Matsuda, N., Satou, J., Nakano, T., \& Ohkuma, H. (2014). Early ambulation produces favorable outcome and nondemential state in aneurysmal subarachnoid hemorrhage patients older than 70 years of age. World Neurosurgery, 81(2), 330-334. doi:10.1016/j.wneu.2012.12.007.

Suarez, J. I. (2015). Diagnosis and management of subarachnoid hemorrhage. Continuum (Minneap Minn), 21(5 Neurocritical Care), 12631287. doi:10.1212/con.0000000000000217.

Tipping, C. J., Harrold, M., Holland, A., Romero, L., Nisbet, T., \& Hodgson, C. L. (2017). The effects of active mobilisation and rehabilitation in ICU on mortality and function: a systematic review. Intensive Care Medicine, 43(2), 171-183. doi:10.1007/s00134-016-4612-0.

Udy, A. A., Vladic, C., Saxby, E. R., Cohen, J., Delaney, A., Flower, O., . Pilcher, D. V. (2017). Subarachnoid hemorrhage patients admitted to intensive care in Australia and New Zealand: A multicenter cohort analysis of in-hospital mortality over 15 years. Critical Care Medicine, 45(2), e138-e145. doi:10.1097/ccm.0000000000002059.

Vergouwen, M. D., Etminan, N., Ilodigwe, D., \& Macdonald, R. L. (2011). Lower incidence of cerebral infarction correlates with improved functional outcome after aneurysmal subarachnoid hemorrhage. Journal of Cerebral Blood Flow and Metabolism, 31(7), 1545-1553. doi:10.1038/ jcbfm.2011.56. 


\section{APPENDIX 1}

\section{SURVEY TO PHYSIOTHERAPISTS}

\section{PART A}

1. Please indicate which geographical location you work in.

- VIC

- TAS

- NSW
- QLD

- WA

- $\mathrm{ACT}$
- SA

- NT

- NZ

2. How many years of experience do you have in the physiotherapy management of patients admitted with acute aSAH?

- $<1$ year

- 1-4 years

- 5-10 years

- $>10$ years

3. Can you estimate approximately how many patients with aSAH you would manage as part of your average monthly caseload?

4. Which aSAH grading scales are used at your hospital?

- The Fisher Scale

- The Modified Fisher Scale

- The Hunt and Hess Scale

- The World Federation of Neurosurgeons Classification (WFNS) Scale

- I am unsure

- Other

5. Please indicate which setting/s best describes where you mainly practice.

- Neurosciences / Neurosurgery Ward

- Dedicated Neurosciences / Neurosurgery ICU

- General ICU with Neurosciences / Neurosurgery casemix

6. Which best describes the hospital facility you work in?

- Public Hospital

- Private Hospital

- Public and private facility combined

7. Please indicate below which of the following mobility items would be a common goal for aSAH patients to achieve before the aneurysm is 'secured' (either by clipping or coiling).

- Sitting up in bed

- Sitting over the edge of the bed

- Passive transfer to sit out of bed (via hoist or patslide)

- Standing

- Step transfer out of bed to chair or commode only

- Marching on the spot

- Walking short distances only (e.g. 10-20m to/from bathroom)

- Ambulation or out of bed mobility without restrictions

- None of the above

- Other 
8. In relation to a patient who is neurologically and cardiovascularly stable, please indicate which day would typically be the first to mobilise a patient after the aneurysm has been 'secured' (either by clipping or coiling).

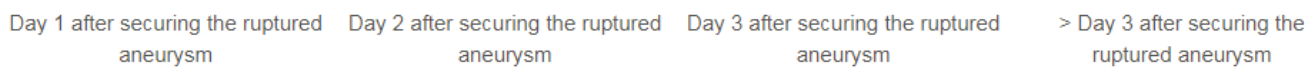

Sitting up in bed
Sitting over the edge of the bed
Passive transfer to sit out of bed
(via hoist / patslide)
chair transfer to sit out of bed in
Ambulation
If > day 3 please indicate the reason:

9. Thinking about neurological precautions to mobility after the ruptured aneurysm has been secured, please indicate the mobility interventions you would be happy to undertake given the following factors.

\begin{tabular}{|c|c|c|c|c|c|c|}
\hline & $\begin{array}{l}\text { Rest in bed / minimal } \\
\text { activity }\end{array}$ & $\begin{array}{l}\text { Sitting up in bed / } \\
\text { head of bed elevated }\end{array}$ & $\begin{array}{l}\text { Sitting over the edge } \\
\text { of the bed }\end{array}$ & $\begin{array}{l}\text { Passive transfer to } \\
\text { chair via hoist or } \\
\text { patslide }\end{array}$ & Step transfer to chair & Ambulatinn \\
\hline $\begin{array}{l}\text { Recent confirmation of } \\
\text { vasospasm on radiographic } \\
\text { images / Transcranial dopplers }\end{array}$ & $\square$ & | & $\square$ & $\square$ & $\square$ & $\square$ \\
\hline $\begin{array}{l}\text { Acute clinical signs of delayed } \\
\text { cerebral ischaemia }\end{array}$ & $\square$ & $\square$ & $\square$ & $\square$ & $\square$ & $\square$ \\
\hline New cerebral infarct & $\square$ & $\square$ & $\square$ & \begin{tabular}{|r} 
\\
\end{tabular} & $\square$ & $\square$ \\
\hline Recent further bleed & $\square$ & $\square$ & $\square$ & $\square$ & $\square$ & $\square$ \\
\hline Low GCS score without sedation & $\square$ & $\square$ & $\square$ & $\square$ & $\square$ & $\square$ \\
\hline Heavily sedated & $\square$ & $\square$ & \begin{tabular}{|l|l} 
\\
\end{tabular} & $\square$ & $\square$ & $\square$ \\
\hline $\begin{array}{l}\text { Severe aSAH (e.g. Fisher 3-4, } \\
\text { WFNS 4-5) }\end{array}$ & $\square$ & $\square$ & $\square$ & $\square$ & $\square$ & $\square$ \\
\hline $\begin{array}{l}\text { High risk vasospasm period (up } \\
\text { to day 14) with no clinical signs }\end{array}$ & $\square$ & $\square$ & $\square$ & $\square$ & $\square$ & $\square$ \\
\hline Nausea and vomiting & $\square$ & $\square$ & $\square$ & $\square$ & $\square$ & $\square$ \\
\hline Recent seizures (e.g. $<4$ hours) & \begin{tabular}{|l|l} 
\\
\end{tabular} & $\square$ & $\square$ & $\square$ & $\square$ & $\square$ \\
\hline cognitive / behavioural issues & \begin{tabular}{|l|l} 
\\
\end{tabular} & $\square$ & $\square$ & $\square$ & $\square$ & $\square$ \\
\hline $\begin{array}{l}\text { Presence of decompressive } \\
\text { craniectomy surgery }\end{array}$ & $\square$ & $\square$ & $\square$ & $\square$ & $\square$ & $\square$ \\
\hline Severe headache & $\square$ & $\square$ & $\square$ & $\square$ & $\square$ & $\square$ \\
\hline Mild headache & $\square$ & $\square$ & $\square$ & $\square$ & $\square$ & $\square$ \\
\hline Invasively mechanically ventilated & $\square$ & $\square$ & \begin{tabular}{|l|l} 
\\
\end{tabular} & $\square$ & $\square$ & \begin{tabular}{|l|}
$\square$ \\
\end{tabular} \\
\hline
\end{tabular}


10. Which of the following parameters do you typically monitor when moving patients into upright positions?

- Systolic blood pressure

- Mean arterial pressure

- Cerebral perfusion pressure

- Heart rate

- Respiratory rate

- $\mathrm{Sp02}$

- Level of consciousness / alertness
- Intracranial pressure

- Upper \&/or lower limb strength

- Cranial nerve function

- Sensation (e.g. numbness, altered sensation)

- Vision

- Headache

- None of the above

- Other

11. In patients with an EVD in situ, would any of the following factors prevent you from clamping the EVD for mobility?

- High amounts of blood draining from the EVD

- Large amounts of CSF being drained per hour (e.g. $>15 \mathrm{ml} / \mathrm{hr}$ )

- Level of EVD recently raised / challenged

- If measured, intracranial pressures of $15-20 \mathrm{mmHg}$

- If measured, intracranial pressures of $>20 \mathrm{mmHg}$

- New signs of neurological deterioration

- Presence of hydrocephalus on brain imaging

- Senior neurosurgeon or intensive care doctor have not given clearance to clamp the EVD

- None of the above

- Other

12. In the setting that you work, are physiotherapists able to clamp the EVD?

- No. Physiotherapists do not clamp the EVD.

- Yes. Physiotherapists clamp the EVD relatively independently.

- Yes. Physiotherapists clamp the EVD, but in close liaison/supervision of nursing staff.

- Only senior / experienced physiotherapists clamp the EVD in close liaison / supervision of nursing staff.

13. For patients who have had a decompressive craniectomy, when can mobility commence?

- Mobility occurs only once a helmet is fitted and able to be worn

- Mobility with a helmet is preferred, but may commence prior if issues such as swelling or wound breakdown prevents its application

- Mobility commences without a helmet

- Other

14. Do you feel that you have the local experience and expertise to answer questions specific to physiotherapy services for aSAH in the ICU environment?

- Yes

- No

15. Do you have an aSAH physiotherapy mobility protocol?

- Yes

- No 
PART B

16. Of the following, which best describes the physiotherapy referral process you have in place for patients admitted to ICU with aSAH?

- Every patient is seen by physiotherapy

- Only patients that are referred by medical staff are seen by physiotherapy

- Only patients that are referred by nursing staff are seen by physiotherapy

- Only patients that are referred by medical and nursing staff are seen by physiotherapy

- Patients are screened by a physiotherapist and seen if indicated

- Other

17. Do patients with aSAH who are in ICU have access to a weekend service?

- Yes

- No

18. If yes, what best describes the weekend service provided?

- Prioritised weekend services according to set criteria, mainly for maintaining respiratory care

- Prioritised weekend service according to set criteria, mainly targeting rehabilitation of patients

- Reduced service over weekend, with normal access to physiotherapy on Saturday at levels similar to the services offered Monday to Friday

- No change in services, same access to physiotherapy on Saturday and Sunday as services offered Monday to Friday

- Other

19. For each mobility item, please indicate the accepted level of authorisation required to undertake the activity before the aneurysm is secured in your setting. You can select more than one option for each mobility item.

If the Neurosurgeon clears this pre-
operatively
Sitting up in bed the Senior Intensive Care Doctor clears this
Sitting over the edge of the bed
Passive transfer to sit out of bed
(via hoist / patslide)
Step transfer to sit out of bed
Marching on the spot
Walking short distances onlition (e.g.
10-20m to/from bathroom)
Ambulation or out of bed activity
without restrictions


20. Thinking about precautions to mobility after the ruptured aneurysm has been secured, please indicate the mobility interventions you would be happy to undertake given the following factors.

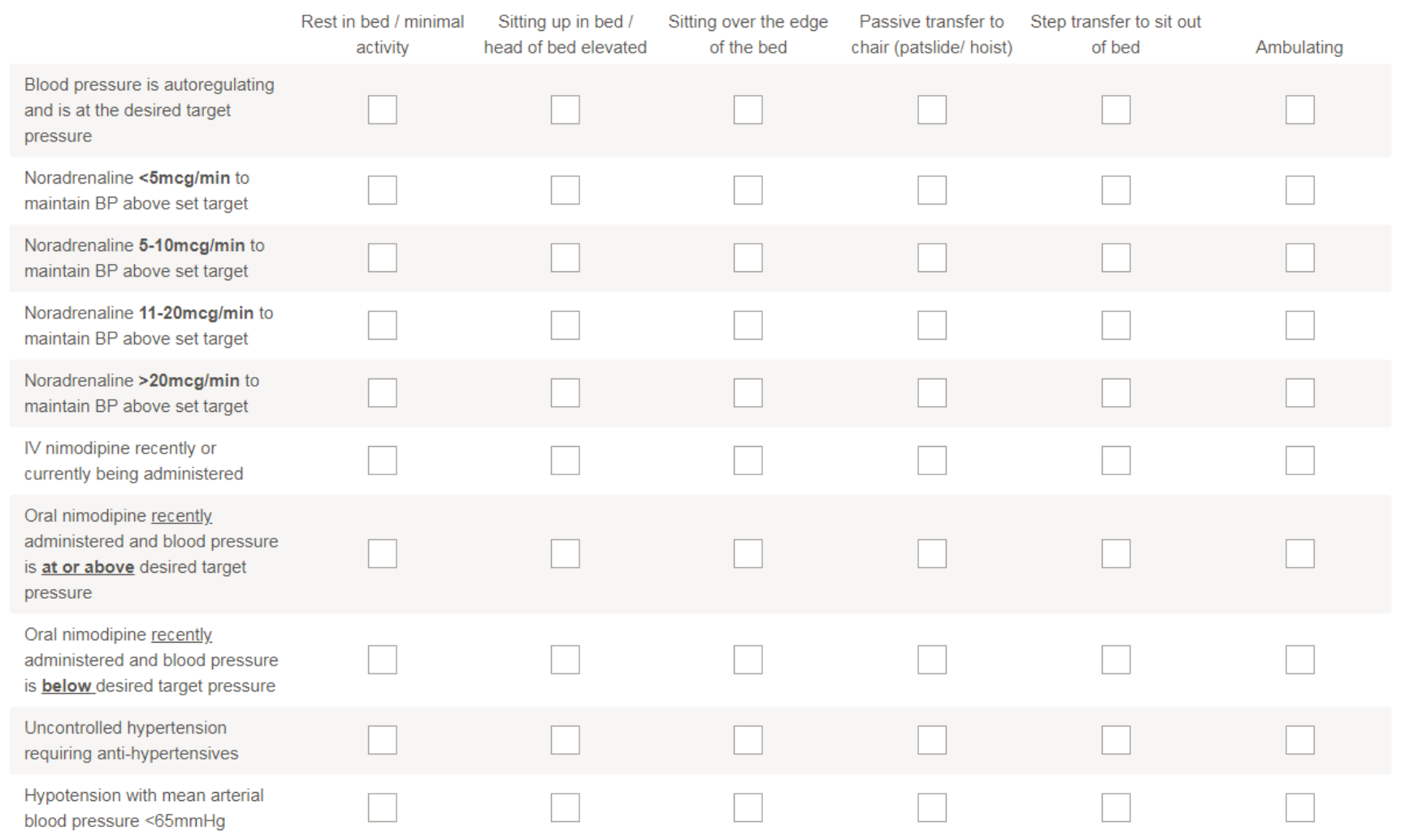

21. For patients with moderate to severe functional limitations, please indicate which physiotherapy interventions are typically provided in the ICU setting?

- Passive joint range of motion exercises

- Active-assisted and/or active range of motion exercises

- Strengthening/resistance exercises

- Motomed cycling

- Functional electrical stimulation (FES) cycling

- Neuromuscular electrical stimulation (NMES)

- Re-positioning in bed (e.g. side-lie positioning, sitting up)

- Sitting up with chair mode e.g. Hill-Rom bed

- Tilt table

- Sitting balance retraining (on the edge of the bed)

- Sit out of bed in a chair either by hoist or patslide

- Standing practice by the bedside with therapist assistance alone

- Standing with a standing machine/hoist or standing frame

- None of the above

- Other

22. For patients that are cardiovascularly and neurologically stable with no signs of respiratory compromise, how many mobility sessions do patients with moderate to severe functional limitations routinely receive per week?

- More than twice a day

- Twice a day 
- Once a day

- Every second day

- Twice a week

- Once a week

- Less than once a week

- Other

23. For patients with mild or no functional limitations please indicate what interventions are typically provided in the ICU setting.

- Passive joint range of motion exercises

- Bed-based active range of motion exercises

- Strengthening/resistance exercises

- Motomed cycling

- Functional electrical stimulation (FES) cycling

- Neuromuscular electrical stimulation (NMES)

- Seated upper limb and lower limb AROM exercises

- Standing upper limb and lower limb AROM exercises

- Standing / dynamic balance practice

- Ambulation practice

- None of the above

- Other

24. For patients that are cardiovascularly and neurologically stable with no signs of respiratory compromise, how many mobility sessions do patients with mild or no functional limitations routinely receive per week?

- More than twice a day

- Twice a day

- Once a day

- Every second day

- Twice a week

- Once a week

- Less than once a week

- Other

25. Please indicate whether you feel access to the following are a frequent barrier to mobilising patients in your unit after aSAH. Frequent is defined as a barrier at least every second day.

- Insufficient staff to assist with mobilising patients with moderate to severe functional limitations

- Insufficient staff to assist with mobilising patients with mild or no functional limitations

- Insufficient staffing to monitor a high falls risk patient when sitting out of bed

- Limited access to appropriate seating/chairs to enable patients with moderate to severe functional limitations to sit out of bed

- Limited access to appropriate seating/chairs to enable patients with mild or no functional limitations to sit out of bed

- Limited access to transferring equipment (e.g. hoists, standing machines or patslides) to enable patients with moderate to severe functional limitations to sit out of bed.

- None of the above

- Other

26. Do you feel that you have the local experience and expertise to answer questions specific to physiotherapy services for aSAH in the neurosurgical ward environment?

- Yes

- No 


\section{PART C}

27. Of the following, which best describes the physiotherapy referral process you have in place for patients admitted to the neurosurgical ward not ICU with aSAH?

- Every patient is seen by physiotherapy

- Only patients that are referred by medical staff are seen by physiotherapy

- Only patients that are referred by nursing staff are seen by physiotherapy

- Only patients that are referred by medical and nursing staff are seen by physiotherapy

- Patients are screened by a physiotherapist and seen if indicated

- Other

28. Do patients with aSAH who are on the neurosurgical wards (not ICU) have access to a weekend service?

- Yes

- No

29. If yes, what best describes the weekend service provided?

- Prioritised weekend services according to set criteria, mainly for maintaining respiratory care

- Prioritised weekend service according to set criteria, mainly targeting rehabilitation of patients

- Reduced service over weekend, with normal access to physiotherapy on Saturday at levels similar to the services offered Monday to Friday

- No change in services, same access to physiotherapy on Saturday and Sunday as services offered Monday to Friday

- Other

30. For each mobility item, please indicate the accepted level of authorisation required to undertake the activity before the aneurysm is secured in your ward setting.

Sitting up in bed
Sitting over the edge of the bed
Passive transfer to sit out of bed
(via patslide / hoist)
Step transfer to sit out of bed
Marching on the spot
Walking short distances only
(e.g. 10-20m to/from bathroom)
Ambulation or out of bed activity
without restrictions


31. Thinking about precautions to mobility after the ruptured aneurysm has been secured, please indicate the mobility interventions you would be happy to undertake.

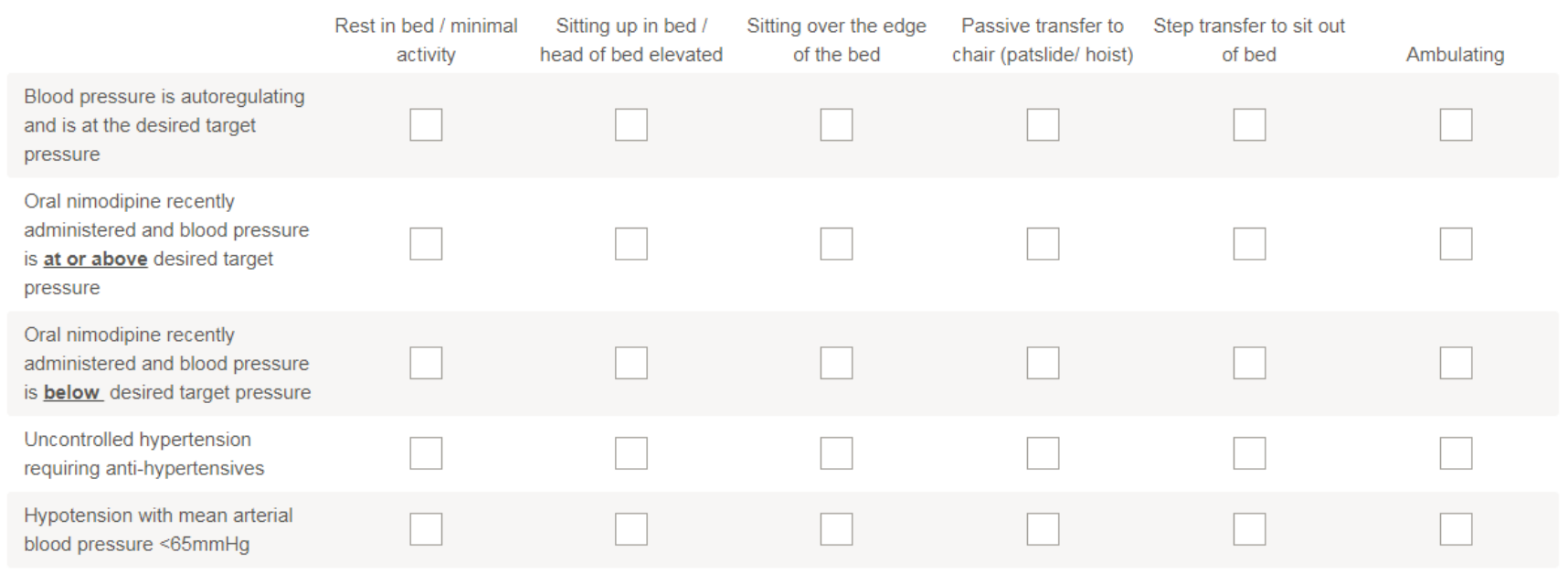

32. For patients with moderate to severe functional limitations, please indicate which physiotherapy interventions are typically provided in the neurosurgical ward not ICU setting?

- Passive joint range of motion exercises

- Active-assisted and/or active range of motion exercises

- Strengthening/resistance exercises

- Motomed cycling

- Functional electrical stimulation (FES) cycling

- Neuromuscular electrical stimulation (NMES)

- Re-positioning in bed (e.g. side-lie positioning, sitting up)

- Sitting up with chair mode e.g. Hill-Rom bed

- Tilt table

- Sitting balance retraining (on the edge of the bed)

- Sit out of bed in a chair either by hoist or patslide

- Standing practice by the bedside with therapist assistance alone

- Standing with a standing machine/hoist or standing frame

- None of the above

- Other

33. For patients that are cardiovascularly and neurologically stable with no signs of respiratory compromise, how many mobility sessions do patients with moderate to severe functional limitations routinely receive per week?

- More than twice a day

- Twice a day

- Once a day

- Every second day

- Twice a week

- Once a week

- Less than once a week

- Other 
34. For patients with mild or no functional limitations please indicate what interventions are typically provided in the neurosurgical ward not ICU setting?

- Passive joint range of motion exercises

- Bed-based active range of motion exercises

- Strengthening/resistance exercises

- Motomed cycling

- Functional electrical stimulation (FES) cycling

- Neuromuscular electrical stimulation (NMES)

- Seated upper limb and lower limb AROM exercises

- Standing upper limb and lower limb AROM exercises

- Standing / dynamic balance practice

- Ambulation practice

- None of the above

- Other

35. For patients that are cardiovascularly and neurologically stable with no signs of respiratory compromise, how many mobility sessions do patients with mild or no functional limitations routinely receive per week?

- More than twice a day

- Twice a day

- Once a day

- Every second day

- Twice a week

- Once a week

- Less than once a week

- Other

36. Please indicate whether you feel access to the following are a frequent barrier to mobilising patients in your unit after aSAH.

- Insufficient staff to assist with mobilising patients with moderate to severe functional limitations

- Insufficient staff to assist with mobilising patients with mild or no functional limitations

- Insufficient staffing to monitor a high falls risk patient when sitting out of bed

- Limited access to appropriate seating/chairs to enable patients with moderate to severe functional limitations to sit out of bed

- Limited access to appropriate seating/chairs to enable patients with mild or no functional limitations to sit out of bed

- Limited access to transferring equipment (e.g. hoists, standing machines or patslides) to enable patients with moderate to severe functional limitations to sit out of bed.

- None of the above

- Other 